\title{
LOS GRUPOS 3X1 COMO EXPERIENCIA DE TRABAJO CON PRODUCTORES YERBATEROS
}

\section{3:1 groups as a work experience with yerba mate producers}

\author{
Gallardo, Roque \\ Gdor. Ferre 2842 AER INTA Virasoro, E mail: gallardo.roque@inta.gob.ar
}

\begin{abstract}
RESUMEN
El trabajo comparte las experiencias sobre el funcionamiento del programa 3x1 (Plan de Renovación de yerbales viejos), articulado entre el Instituto de Tecnología Agropecuaria (INTA) y el Instituto Nacional de la Yerba Mate (INYM), con desarrollo en la Agencia de Extensión Rural INTA Virasoro. A través de la articulación en terreno de los diferentes actores, se logró dar cumplimiento satisfactorio al objetivo principal del citado programa, la instalación de una parcela de plantación de yerba mate desde una intervención socioproductiva innovadora y sostenible. Ello, a través de una metodología participativa con fuerte implicancia de todos los integrantes de esta iniciativa que incluyó la implementación de diversas dinámicas grupales destinadas al relevamiento colectivo permanente sobre la práctica y la incorporación de nuevos aportes. Los resultados obtenidos muestran la participación activa de 26 productores en las experiencias, incluyendo la asistencia técnica a partir de 18 instancias de formación y capacitación en temas específicos de la producción yerbatera, y la plantación de 57200 plantas de yerba mate de origen policlonal de INTA con técnicas de manejo sostenible.
\end{abstract}

Palabras clave: experiencias socioproductivas, articulación, grupos, programa, yerba mate.

\begin{abstract}
This work shares experiences about $3 \times 1$ (plan for the renewal of old yerba mate plantation) program operation, articulated between the Institute of Agricultural Technology (INTA) and the National Institute of Yerba Mate (INYM), which is put into practice at the Rural Extension Agency INTA Virasoro. Through the articulation in the field of the different participants, it was possible to satisfactorily fulfill the main objective of the mentioned program, building on a yerba mate plantation out of an innovative and sustainable socio-productive intervention. This was owing to a participatory working methodology with strong involvement of all members of this initiative. This proposal included the implementation of various group dynamics aimed at the permanent collective survey of the practice and the incorporation of new contributions. The results obtained showed the active participation of 26 producers in these experiences, including technical assistance from 18 training and instruction sessions as regards yerba mate production and the planting of 57200 polyclonal origin yerba mate plants from INTA with sustainable management techniques.
\end{abstract}

Key words: socio-productive experiences, articulation, groups, program, yerba mate.

Recibido: 02/jun/2020. Aceptado: 25/jul/2020 


\section{INTRODUCCIÓN}

En la región yerbatera de la República Argentina, que corresponde al NE de Corrientes y la provincia de Misiones, se encuentran presentes diferentes estratos de productores con una marcada fragmentación del sector. En ambas provincias, el sector que representa más de un $80 \%$ del área se corresponde con productores que poseen superficies inferiores a 25 hectáreas, tal como lo expresa Gortari (2013). En su mayoría, estos productores pequeños se caracterizan por deficiencias en el manejo del sistema productivo, con excesos de laboreos en los suelos, problemas de compactación, disminución de los rendimientos promedios de las chacras y, en muchos casos, bajas densidades en sus plantaciones, hasta incluso yerbales de avanzada edad.

En la región yerbatera de la República Argentina, que corresponde al NE de Corrientes y la provincia de Misiones, se encuentran presentes diferentes estratos de productores con una marcada fragmentación del sector. En ambas provincias, el sector que representa más de un $80 \%$ del área se corresponde con productores que poseen superficies inferiores a 25 hectáreas, tal como lo expresa Gortari (2013). En su mayoría, estos productores pequeños se caracterizan por deficiencias en el manejo del sistema productivo, con excesos de laboreos en los suelos, problemas de compactación, disminución de los rendimientos promedios de las chacras y, en muchos casos, bajas densidades en sus plantaciones, hasta incluso yerbales de avanzada edad.

Considerando esta situación generalizada en el sector más vulnerable, desde los organismos públicos y privados vinculados a la cadena yerbatera, se confecciona el Programa de Renovación de Viejos Yerbales. Este, se inscribe dentro de las acciones que ejecuta el Instituto Nacional de la Yerba Mate, a través del departamento técnico de dicho Instituto. Así, por medio de la articulación con el INTA y a través de la metodología del Programa de Cambio Rural, se lanza el plan de reconversión en el año 2012, que está en consonancia con el Plan Estratégico para el sector yerbatero (INYM, 2020).

El mismo es conocido como Tres por Uno, está destinado a agricultores de hasta 10 hectáreas de yerba mate con superficie degradada. Apunta a lograr que el productor obtenga en una hectárea, mejor rendimiento que en tres hectáreas, mediante técnicas conservacionistas en suelo, la implantación de plantas mejoradas genéticamente, y que al cabo de cinco años de iniciado el proceso, destine tres hectáreas de yerbales degradados a otra actividad agropecuaria o forestal. Este programa de intervención, se ejecuta con financiación del Instituto Nacional de la Yerba Mate (INYM) y la participación del Instituto Nacional de Tecnología Agropecuaria (INTA) a través de las distintas Agencias de Extensión Rural que se encuentran en la zona productora de yerba mate. De la misma manera, el programa prevé la contratación de técnicos privados que puedan asistir y monitorear en forma conjunta con el técnico de INTA las actividades en los territorios.

En esta línea de trabajo, desde la Agencia de Extensión Rural (AER) de INTA, en la localidad de Gobernador Virasoro, nos súmanos al programa de reconversión yerbatera, promoviendo acciones en forma conjunta con el INYM y las asociaciones de productores. Se estableció una zona de aplicación del programa dentro del área de influencia de la AER, relevando a los productores que estaban dentro del perfil señalado. Este trabajo inicial, se constituyó en forma conjunta con las asociaciones de productores de la región.

A continuación, en la figura 1, se muestra la ubicación de los integrantes de los tres grupos del convenio entre el INTA y el INYM, en el marco del programa 3x1, para la AER INTA Virasoro, que se encuentran ubicados entre las rutas provinciales nro. 68 que une la localidad de Colonia Garavi con la ciudad de Gobernador Virasoro; las ruta provincial nro. 37, que se extiende hacia el Este un culmina en la localidad de Garruchos, sobre la margen del rio Uruguay.

El objetivo principal del programa consistió en instalar una parcela de plantación de yerba mate nueva, como experiencia innovadora a partir del programa de reconversión $3 \times 1$, con una metodología de intervención participativa. Esto último, desde la implementación de diversas dinámicas grupales para relevar y poder actuar sobre las diversas situaciones presentadas por parte de los productores participantes. Además del objetivo principal del programa, se promovieron los siguientes objetivos específicos:

- Promover el trabajo grupal de pequeños productores yerbateros

- Mejorar la aplicación de buenas prácticas agrícolas en el manejo de los yerbales

- Articular con otros organismos del estado. 


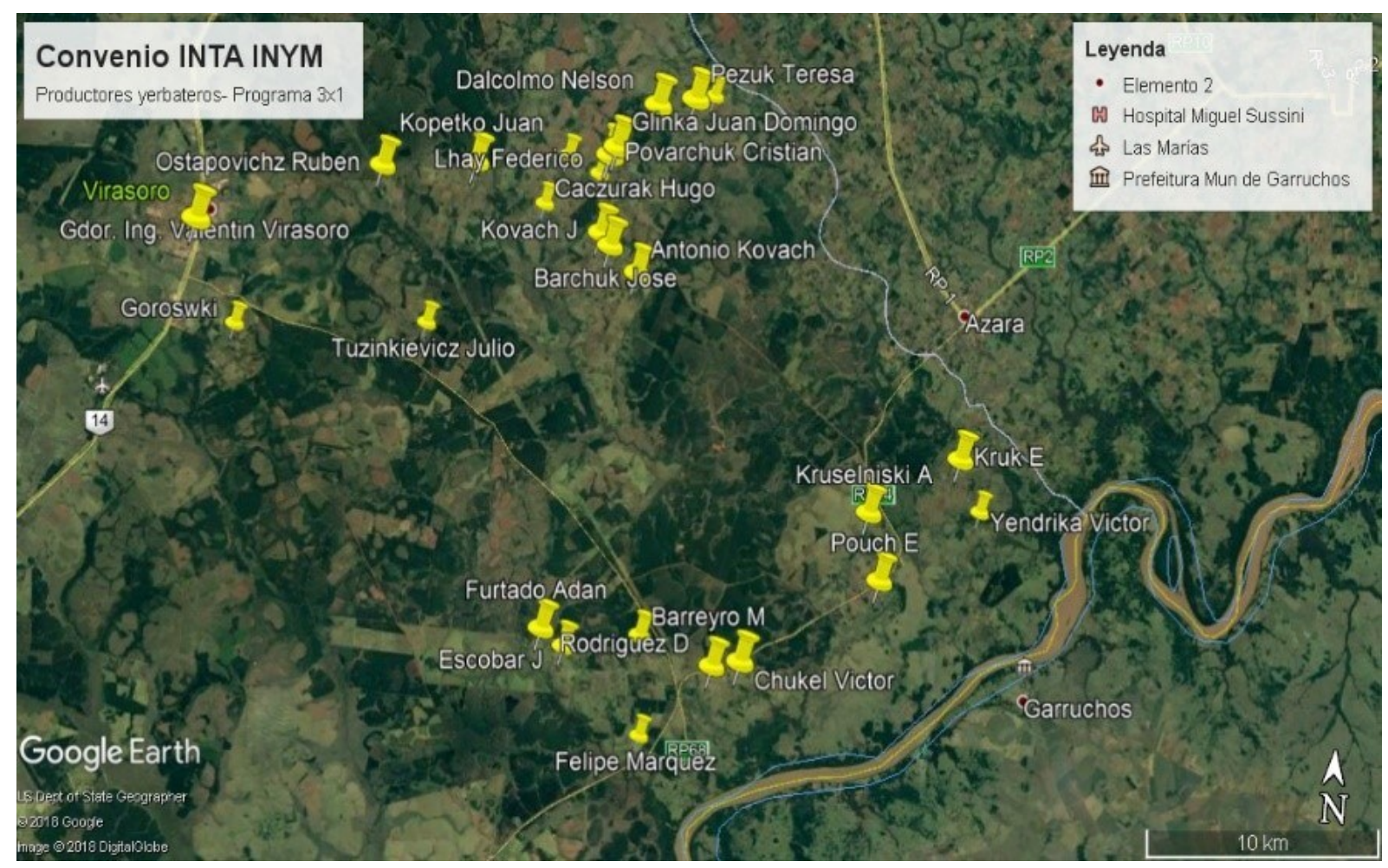

Figura 1. Mapa de ubicación de chacras convenio INTA-INYM

\section{METODOLOGÍA}

En el marco de una metodología participativa, el programa despliega diversas estrategias que incluyen el trabajo grupal, lo cual implica un involucramiento activo de los productores junto al equipo técnico. Buscando a través de la construcción y comprensión del discurso ( productores y técnicos), lo que se quiere decir que debe ser interpretado por uno y por otro de manera coincidente, además de analizar el sentido y las condiciones para comprender el mensaje (Darre, 1996). Siendo esto importante para construir de manera participativa las tareas que se realizan y por consecuencia el camino a seguir.

A través de reuniones de sensibilización se pudieron conformar los grupos de trabajo elaborándose a través de los diversos talleres, un diagnóstico inicial general y de cada integrante tal como plantea Geilfus, (2000). De esta manera, se logró conocer el panorama general de los grupos de trabajo y las diferentes situaciones individuales presentes en los yerbateros. Utilizando la técnica de "Árbol de problemas" como señalan Ortegón et al. (2005), se pudieron relevar las situaciones problemáticas presentes, acordar los objetivos de trabajo y, con una priorización de las actividades, se definieron las propuestas de acción para cada grupo de trabajo. En estos espacios de interacciones se van construyendo de manera compartida el camino donde se quiere llegar, tal como lo expresa Mata (1994).

Se elaboró en forma conjunta un cronograma de trabajo de manera grupal e individual, en los cuales se incluyó cuestiones relacionadas a exigencias del programa como preparación de lotes a implantar, control y manejo de las adversidades, implantación de cubiertas verdes de verano e invierno y otras actividades productivas que planteaban los productores como manejo de las plantaciones de yerba mate.

Las reuniones grupales mensuales, donde participaban todos los integrantes, fueron los momentos en los cuales se realizaron capacitaciones teóricas y prácticas en las explotaciones de los beneficiarios del 3x1. Durante el desarrollo de las actividades, se incluyó un método de evaluación en proceso, a través de la relación de dialogo democrático entre educador- extensionista y educando- productor, como plantean Tommasino y De Hegedüs (2006). Esto sirvió para analizar el rumbo del grupo en cuanto a cuestiones tales como la correspondencia entre las diferentes actividades propuestas a partir de los diagnósticos iniciales con lo realizado hasta ese 
momento, considerar la necesidad de generar reorientaciones atento a factores externos al conjunto de los productores, entre otras, buscando siempre y en acuerdo con (Freire, 1985) " escuchar y obrar" de manera crítica para llegar entre todos a construir y compartir el conocimiento.

Asimismo, como complemento de estas actividades se realizaron visitas individuales a los lotes implantados, en las cuales se pudieron constatar con el productor el estado de situación de los mismos como así también todo el sistema productivo. De todo esto se puede aseverar que es importante problematizar con situaciones reales que está viviendo el productor agropecuario, de manera de poder visualizarlas y actuar desde la cítrica y la posterior acción conjunta de técnicos y productores.

\section{RESULTADOS OBTENIDOS}

Se conformaron tres Grupos de yerbateros, dentro del Convenio INTA INYM Programa Reconversión Yerbatera, con 26 productores asistidos técnicamente y 26 hectáreas de yerba mate cultivadas nuevas. Ello, representando un total incorporado de 57.200 plantas, de material genético certificado y origen policlonal de INTA. En la Figura 2 se muestra una implantación realizada en curvas de nivel.

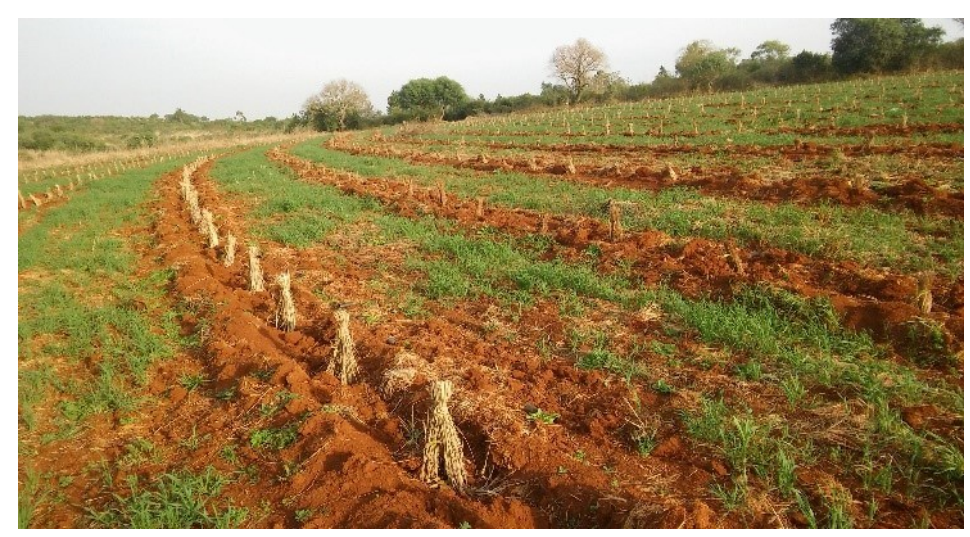

Figura 2. Yerbal implantado en curva de nivel Zona Colonia Unión. Gallar do Roque

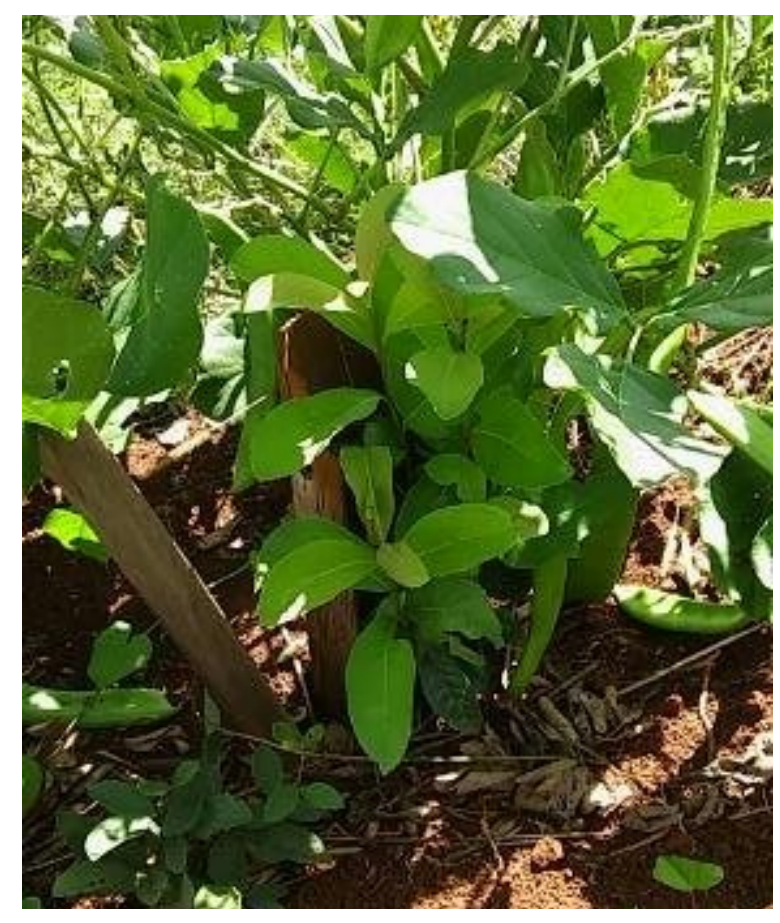

Figura 3. Por oto Sable ( Canavalia ensiformis) en lineo de implantación de yerba mate. Gallardo Roque 
Como resultado de las actividades de sensibilización, respecto al cuidado del suelo, se logró un laboreo sobre el trazado para que las plantaciones se realicen en curvas de nivel, con un manejo sustentable tanto del suelo como así también de las plantas. Esto posibilitó también una excelente preparación de los lotes de implantación utilizando subsolados profundos, a más de 60 centímetros de profundidad, siendo el verano la época más conveniente. Además, se promovió la utilización de cubiertas verdes de invierno y verano en los lotes de yerba mate implantados, como se observa en las figuras 2 y 3 .

Los primeros lotes implantados con este programa en el área de influencia de la AER, en las intervenciones iniciales destinadas a la formación de plantas, alcanzaron un rendimiento promedio por planta, (en poda de formación), de $5 \mathrm{~kg}$ de material de cosecha que va al secadero (ramas finas y hojas), lo que estima un total de aproximadamente 10.000 kilogramos por hectárea. Dicho resultado, se puede considerar un excelente rendimiento, si tomamos en cuenta que la media de la provincia de Corrientes esta alrededor de los 5.300 kilogramos, lo cual manifiesta un potencial interesante a partir de las plantaciones establecidas por el programa.

La Articulación público-privada (INTA-INYM- Productores-Técnico privados), demostró ser una estrategia clave para lograr el desarrollo de las diferentes actividades en simultaneidad con el fortalecimiento del abordaje territorial de la AER Virasoro. De la misma forma, se pudo trabajar en forma conjunta con el municipio de Virasoro en la preparación de los lotes de implantación de yerba mate, como así también en el seguimiento de los lotes implantados en la zona de colonia Unión, San Justo, Garabi, Garruchos.

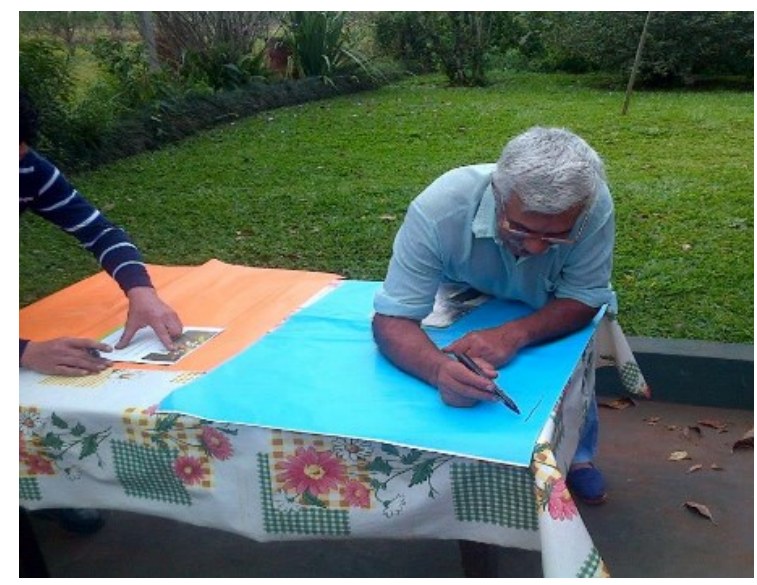

Figura 4. Taller Mapeo de Chacras. Gallardo Roque.

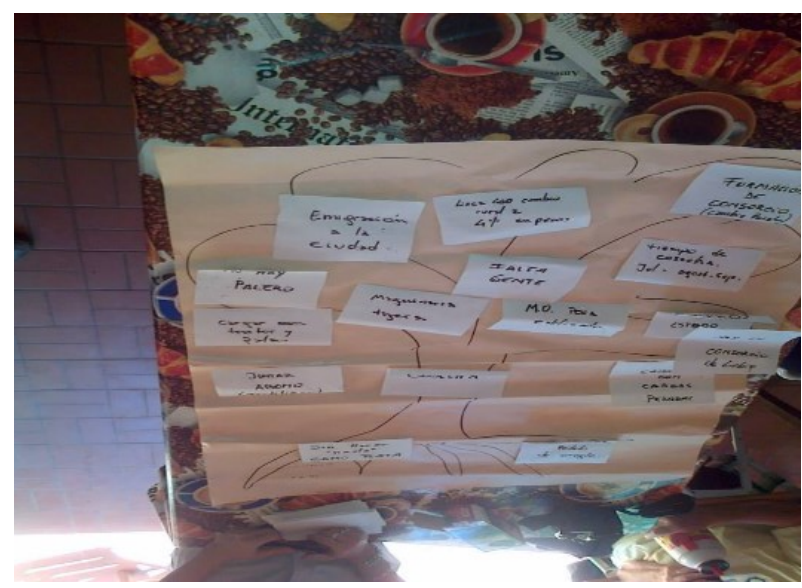

Figura 5.Taller Elaboración de Árbol de problemas realizado por los productores. Gallardo Roque.

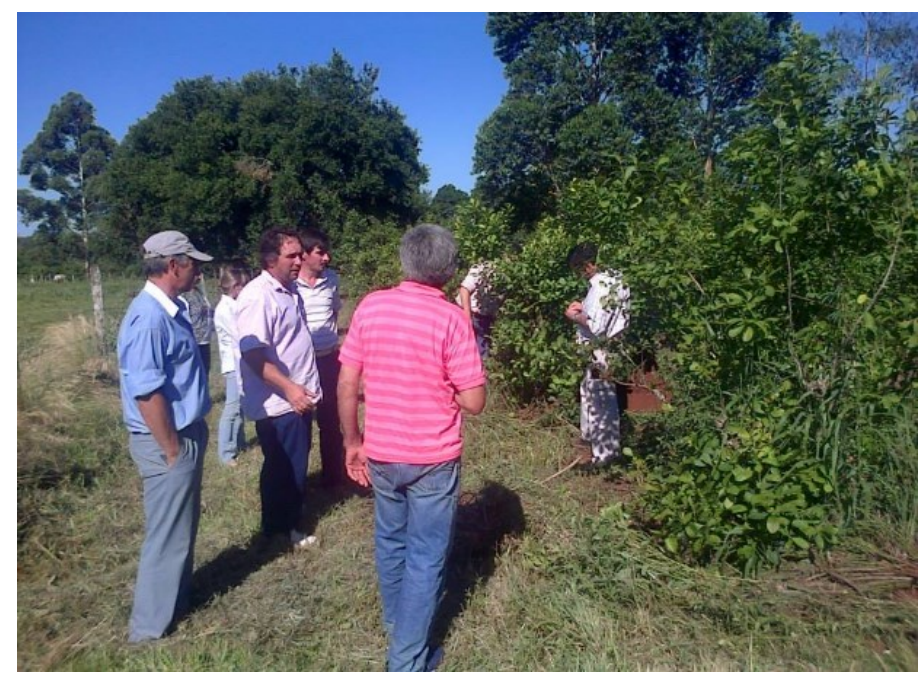

Figura 6. Capacitación en poda de yer ba mate. Gallardo Roque. 
Se promovieron más de 18 instancias de capacitación en diferentes temáticas que surgieron de las reuniones y talleres organizados con los diversos grupos, entre las cuales se destacaron: mapeos y elaboración de árbol de problemas de las chacras, como parte del trabajo de campo situado en el propio territorio (figuras 4 y 5), el trazado de curvas de nivel; la implantación de cultivos; el manejo y utilización de las cubiertas verdes; el desarrollo de estrategias de fertilización química y orgánica en lotes de yerba mate nuevos y en producción; el manejo de las diversas malezas y otras adversidades; las capacitaciones en Podas de formación en plantaciones nuevas y en producción (figura 6).

\section{CONSIDERACIONES FINALES}

La implementación del programa permitió desplegar con éxito no sólo los objetivos propuestos, sino que posibilita una sostenibilidad en cuanto a la continuidad de la metodología de trabajo, en las propias experiencias en curso, así como en nuevas intervenciones. El desarrollo de las capacitaciones y el acompañamiento en la dinámica de grupos, lograron materializar cuestiones relevantes para la mejora del sistema productivo y, consecuentemente, las condiciones de los propios productores. A través de un correcto manejo y desde un enfoque sistémico, se pudo trabajar integralmente sobre los componentes suelo y planta, así como las adecuadas cosechas. La instalación de los lotes de yerba nueva se considera lograda en su totalidad. Como producto del trabajo grupal generado desde el programa, los productores incorporaron en la práctica propia de manejo el concepto de sistema productivo.

La articulación con otros organismos e instituciones del Estado, así como con el sector privado asociado al sistema productivo en cuestión, demostró ser fundamental para implementar con éxito el programa 3x1 en la zona de la AER Virasoro. Ello, en función de la naturaleza socioproductiva sostenible que define al programa y que comprende las dimensiones de lo asociativo y territorial. Cabe destacar, en tal sentido, que el apoyo de los municipios donde se radicaron las experiencias es destacable al momento de la realización de los trabajos de campo (preparación de suelos, riegos, entre otras cosas).

\section{BIBLIOGRAFÍA}

Darre, J. (1996). La invención de practicas en la agricultura - Difusión y Producción Local de Conocimientos. Paris: Ed. Karthala.

Freire, P. F. (1985). Por uma pedagogia da pergunta. Rio de Janiero: paz y Tierra. Coleção EDUCAÇÃO E COMUNICAÇÃO Vol. 15.

Geilfus, F. (2000). 80 Herramientas para el Desarrollo Participativo: diagnóstico, planificación, monitoreo, evaluación. IICA-GTZ, San Salvador, El Salvador. 208 pp.

Gortari, J. (2013). Concentración y diferenciación social en la economía regional yerbatera. Voces en el Fénix: 52-59.

INYM. (2020). www.inym.org.ar. Obtenido de https://www.inym.org.ar/operador/plan-estrategico/ $(21 / 07 / 2020)$

Mata, M.C. (1994). Nociones para pensar la comunicación y la cultura masiva. Cur so de especialización Educación para la comunicación. Centro de Comunicación educativa La Crujía.

Ortegón, E., Pacheco, J.F. y Prieto, A. (2005). Metodología del marco lógico. Para la planificación, el seguimiento y la evaluación de proyectos y programas. CEPAL. Serie Manuales $\mathrm{N}^{\circ} 42$.

Tommasino, H. y De Hegedüs P. (2006). Extensión, reflexiones para la intervención en el medio urbano y rural. Montevideo Uruguay: Universidad de la Republica Facultad de Agronomía. 